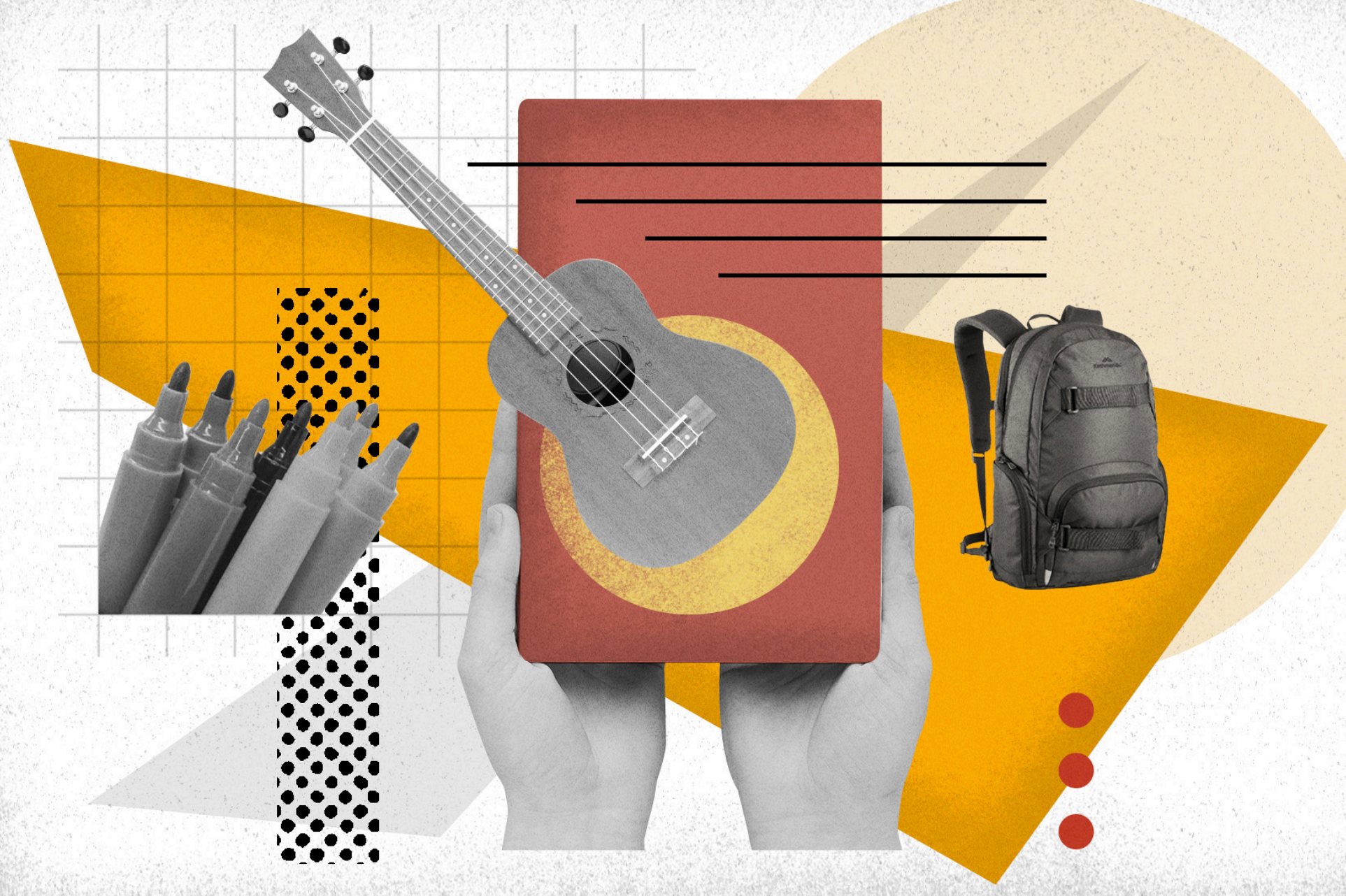

\title{
Escenarios de la experiencia estética en el aprendizaje
}

SCENES OF LEARNING'S AESTHETICS EXPERIENCE CÉNARIOS DA EXPERIENCIA ESTÉTICA EM O APRENDIZAGEM Luis Alfonso Rubio Pardo 


\section{Luis Alfonso Rubio Pardo}

- Colegio La Palestina (IED). Magíster en Educación, Universidad Distrital Francisco José de Caldas. ORCID:https://orcid.org/0000-0001-6915-4276; correo electrónico: carajopolis@gmail.com

Citar artículo como:

Rubio, L. (2020, Julio-Diciembre). Escenarios de la experiencia estética en el aprendizaje. Revista Educación y Ciudad, No. 39, pp. 97-107. //doi org/10.36737/01230425.n39.2020.2336

\section{DOI: https://doi.org/10.36737/01230425.n39.2020.2336}

Fecha de recepción: 2 de febrero de 2020 / Fecha de aprobación: 1 de julio de 2020

\section{Resumen}

El presente artículo describe y examina algunas experiencias escolares vividas durante la realización de un trabajo que propone la vinculación de la música y la literatura, entendiendo dicha relación como elemento esencial para la enseñanza de la asignatura de lengua castellana, procurando forjar un espacio para el surgimiento de una experiencia estética que reúna al estudiante, al maestro y a la escuela en un proceso formativo cada vez más significativo e integral.

Palabras clave: Experiencia estética, música y literatura, sentidos éticos, educación estética, polifonía en la escuela.

\section{Abstract}

The present article intents to describe and examine some school experiences lived during the execution of a work that suggests the link of music and literature as an essential element to the teaching of the Spanish Language. The aim is to create a space to the emergence of an aesthetic experience that brings together the student, the teacher in an educational process evermore significant and comprehensive.

Keywords: Aesthetic experience, music and literature, ethic sense, aesthetic education, school polyphony.

\section{Resumo}

O presente artigo busca descrever e examinar algumas experiencias escolares vividas durante la realização de un trabalho que propõe a vinculação da música e da literatura como elemento essêncial para o ensino de lingua castelhana; buscando criar um espaço para o surgimento de uma experiência que aproxima o estudante, o professor e à escola em um processo formativo cada vez mais significativo e integral.

Palavras-chave: Experiencia estética, música e literatura, sentidos éticos, educação estética, polifonia escolar. 


\section{Acústica de una vivencia}

\section{en el aula}

U na de las características más interesantes de la labor docente es el aspecto vivencial que se construye tejiendo los tiempos dentro de la clase. Las sesiones en el aula, por más que se premediten, son enriquecidas por una especie de combustión vital que ofrece ritmos impredecibles en mayor o menor medida.

El tiempo de trabajo generado en la escuela perfila un cauce que va mucho más allá de episodios profesionales, convirtiéndose en presencia acogida en el espacio personal. Las clases pasan a ser vivencias que tienen cabida en una historia íntima, por eso algunas de ellas quedan marcadas y nuestros recuerdos, como maestros, activan constantemente dichas experiencias.

Al momento de escribir estas líneas viene a la mente una tarde muy calurosa, allá, en el tiempo, se extiende por algún salón de clase del occidente bogotano, durante los irregulares períodos veraniegos que nos brinda la ciudad. El sol se apresuraba inclemente a través de la amplitud de los ventanales que cumplían, ahora en exceso, su función de permitir la entrada de luz. El espacio que compartíamos con los estudiantes se tornaba cada vez más sofocante y se sumergía en la densidad reverberante. Las condiciones parecían cada vez más adversas con el aire pesado y la impertinencia de la transpiración.

No se hizo esperar el guiño de los ecos musicales y las palabras, el sonido de Los prisioneros, banda de rock chilena que dio vida a El baile de los que sobran, en cuyas letras escuchamos una descripción que brinda también su propio dibujo de "esa cosa llamada educación", en donde "había tanto sol sobre las cabezas". El juego de ecos no se detuvo y, tal vez, junto al espesor del clima, generó nuevas visiones y apariciones acústicas enviadas por el sol, desenvueltas en desiertos y playas argelinas, "Staring at the sea, staring at the sun", porque ahora era Killing an arab, delirante composición de The Cure que surgía entre los rincones, como espejismo sahariano.

De esta manera, se sucedían los comentarios y charlas sobre la canícula que se convertía vertiginosamente en evocaciones musicales. Comenzó entonces una pesquisa acerca de las letras de la canción, proponiendo a su vez la creación de un cuadro de campos semánticos en donde se exploraban las palabras relacionadas con este ámbito de sol, calor, arena y mar; llegando, tras aquel ejercicio participativo, al terreno propio de la literatura universal. Así encontramos a El extranjero, célebre novela de Camus que inspiró directamente la letra de The Cure, que en su momento conformó una inesperada plataforma para llegar, junto a los estudiantes, al espacio de los libros y la lectura de una obra magnífica.

Tomando en cuenta esta sencilla pero significativa vivencia dentro del aula, en una jornada cualquiera de las que tenemos como maestros, es posible percibir que existe una especie de latencia en cada una de las clases, la cual agolpa afluentes más o menos insospechados en búsqueda de una forma de florecer en la dinámica educativa. La situación coyuntural y su vivencia generaron, en este caso, series de posibilidades que se articulaban en secuencias más cercanas a la espontaneidad y a 
la confianza, en las asociaciones que fluctuaban durante la sesión.

Pero, siendo consecuentes, es justo analizar ese espacio que admite la espontaneidad y la fluctuación entre los participantes, de manera que no quede reducido a consideraciones anecdóticas o a simples episodios curiosos que se podrían esterilizar en su volatilidad. El carácter que rescatamos de una vivencia de este tipo también demanda atención a sus fecundas posibilidades de construcción y orientación pedagógica, no para aprisionarlo en el rigor de una estructura, sino para pulir e impulsar sus derroteros y su energía. Se trata, más bien, de aprender a encontrar cierto estado propicio para ir a la deriva, pues, como anota Max Neef:

Quien quiere comprender, quien quiere descubrir los mundos paralelos, quien quiere poder trabajar como ser completo, con lenguajes y silencios, quien quiere vivir una vida que sea realmente aventura, debe aprender a derivar pero en estado de alerta [...] Derivar en estado de alerta no es lo mismo, por cierto, que dejarse llevar por el río o la corriente (1992, p.7).

Por ello, es necesario definir líneas y recorridos que se encuentren con estas formas del desarrollo de la enseñanza, cuya vivencia cobra tanta importancia para la escuela. Así, en estas páginas se procura exponer una serie de cuestionamientos e iniciativas que surgen a partir de considerar experiencias como la narrada, en el contexto de la elaboración del trabajo de grado llamado: "La experiencia estética con sentidos éticos a través de la vinculación de la literatura y la música en la enseñanza de la lengua castellana -Una propuesta pedagógica desarrollada con los estudiantes de 902 del Colegio La Palestina-"; el cual, durante su desarrollo, puso en marcha una propuesta que facilitó la configuración de diversas experiencias, cuyas características se tendrá la oportunidad de comentar.

Las propiedades de ciertos momentos en la escuela conservan entonces una importancia vívida en la práctica docente, en donde, como en el ejemplo narrado, vemos desdoblar, desde una condición climática, un camino hacia la vinculación de la música con el aprendizaje, el cual apunta hacia el mundo del lenguaje y la literatura. Este carácter está inscrito en las variaciones de las experiencias pedagógicas que se llevaron a cabo en la propuesta, y su análisis trajo consigo apreciaciones y reconfiguraciones que cimentaron esquemas cada vez más amplios en torno a la enseñanza.

No solo se habla de la asociación espontánea, que puede ocurrir a partir de situaciones fortuitas más o menos proclives a ser articuladas dentro de las temáticas de una asignatura, se trata, ante todo, de encontrar una pauta y unas señales que permitan orientar, abrir el camino latente en tales momentos y desentrañar las oportunidades que alberga para el acto pedagógico.

\section{El bosquejo progresivo de una propuesta pedagógica}

La idea que se persigue pregunta, además de lo planteado, también por los contenidos propios de nuestras asignaturas (en este caso particular, Lengua Castellana), con la intención de encontrar su potencia y valor real. Entendiendo que el cuestionamiento de lo que llamamos tradicional debe llevarse a cabo cuidadosamente, procurando movimientos constructivos que activen y desactiven lo que es justo para un desarrollo cada vez más extenso de la enseñanza. 
La inquietud por los contenidos tradicionales en la escuela ha sido un problema ya identificado con frecuencia, sin embargo, parece un atolladero difícilmente eludible. Entre otros, como editores de un compendio que trabaja el tema, Lomas y Jurado (2015), plantean distintas preguntas frente al carácter tradicional de los libros de texto, entre ellas, vale la pena destacar la propuesta desde el texto de Bombini, quien, citando a Chevellard, resalta que el conocimiento escolar es un "conocimiento sin origen, sin historia ni historicidad, oculta su carácter arbitrario, sus rasgos polémicos y sus matices como prescripción curricular en los documentos oficiales" (Lomas y Jurado, 2015, p.22).

Ahora bien, aunque es preciso dar un espacio considerable a tal discusión, que también incluye el problema de la crisis cada vez más aguda de la escuela, los esquemas aquí postulados no niegan las virtudes de los textos y temas que han existido tradicionalmente, los cuales, además, organizan muchos currículos; más bien, pretenden buscar alternativas de fondo para reencontrar las posibilidades del valor estético que detentan y su interpelación a sentidos fundamentales en la formación de un ser humano.

Así, en este punto es pertinente exponer, aunque sea someramente (pues no es el objetivo principal del presente caso), el esquema insinuado anteriormente. Es importante recordar que se trata de una propuesta cuyo interés es poner en marcha un proceso de aprendizaje que vincule los valores estéticos, particularmente los de la música, con los saberes propios de la asignatura de Lengua Castellana. La siguiente imagen, que podemos denominar Bosquejo orbital de acción, permite ilustrar el proceso señalado:

Figura 1. Bosquejo orbital de acción

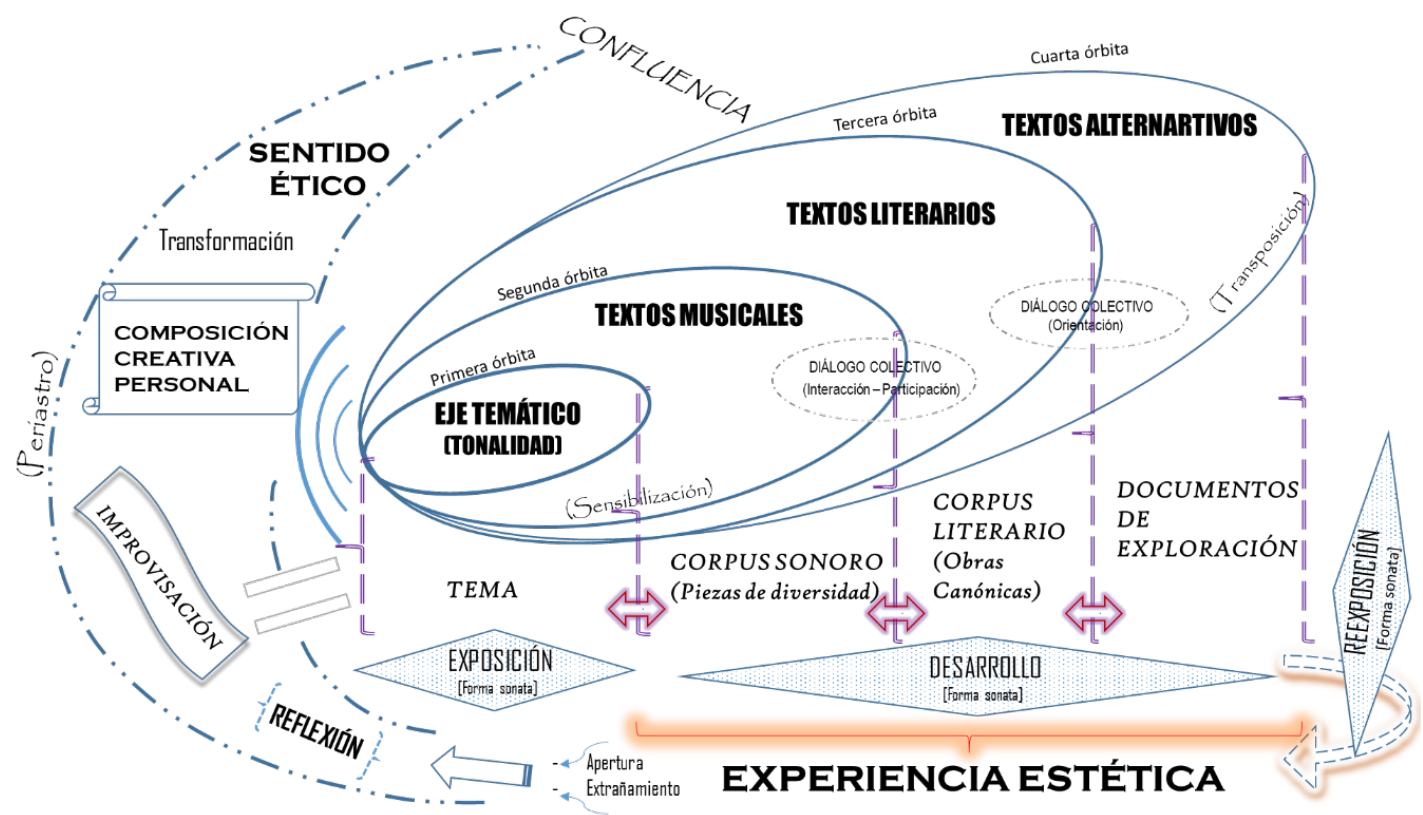




\section{El carácter existencial de la escuela y la implicación del maestro}

\section{Desde una metodología como heurística y cuestionamiento}

Al momento mismo de comenzar a hablar de la propuesta pedagógica y de los esquemas puestos en práctica a lo largo del trabajo, se trataron las primeras acotaciones importantes respecto de la experiencia, pues la sola construcción de un método que trata aspectos cada vez más diversos, y cuya dinámica se inscribe poco a poco en el paradigma de la complejidad, incluye la vivencia del maestro, que se ve inmersa en los giros y elasticidad de esta propuesta que, inicialmente, se destinó a los estudiantes y a la práctica en el aula. Estas observaciones conducen necesariamente a subrayar, junto a Bárcena (2000), que: "La auténtica experiencia del aprender no cobra vida solo en ese marco institucional deliberadamente pedagógico. Es un proceso existencial" (p.13).

Así pues, la experiencia inicia desde la construcción de un bosquejo o un esquema; esto hace necesario inquirir por las formas propias del método, lo cual acarrea inquietudes cada vez más sensibles frente a lo experimentado por el maestro. La elaboración de una metodología propia implica sendos cuestionamientos que, en este caso, llevaron a una pregunta corta y densa, plantada en el origen de las actividades escolares: ¿Por qué?; ¿̇cuál es la razón para iniciar una clase?; ¿por qué debemos dedicar tiempo y espacio a un tema determinado? De la mano con estos interrogantes, escuchando, tal vez susurrantes y refundidos en el interior, aparecen otras preguntas que interpelan, cuestionando: ¿Por qué nuestra labor?; ¿por qué hacemos lo que hacemos?; ¿por qué estamos es este lugar que llamamos escuela y qué significa esto?; ¿por qué estamos aquí?

Tales preguntas llevan a percatarse de que, a la larga, se trata de una forma que toma la angustia en la experiencia docente, y es justo dar su lugar a la importancia de reconocer esta sensación, que puede embargarnos en distintos grados y de distintas maneras. Sin embargo, también se debe recalcar que el contexto educativo permite mirar aquella angustia desde otra óptica, yendo más allá de su percepción como algo negativo, que se debe superar o suprimir, para reconfigurarla bajo otro ángulo, como germen de acción y creación.

En este sentido, Harold Bloom (1995) se permite destacar otro tono en esa situación de ansiedad, planteando la fecundidad de la angustia como influencia para el escritor: "La estética y la agonística son una sola cosa (...) finalmente la gran obra que uno consigue escribir es la misma angustia" ( $p$. 18). De igual forma, la profesora Greene considera este espacio angustioso desde otra perspectiva, enunciando que:

\footnotetext{
La angustia es el camino en que la libertad se revela a sí misma. Es la expresión del continuo deseo de conclusión ( sin ninguna garantía de que la conclusión buscada sea estimable cuando se alcance). El hastío es el modo en que el miedo a la nada y a la indiferencia se revela a la conciencia (Citada en Larrosa, 1995, p.100).
}

Tales apreciaciones hablan de una forma de reconocerse como maestro a partir del propio ejercicio, el cual, desde los cimientos, llama a una constante reflexión, comenzando por la preparación de ma- 
teriales o métodos, la labor impele a un ejercicio del pensamiento y de múltiples cuestionamientos, comprometiendo también la sensibilidad humana inherente al maestro. El rol de profesor puede permitirse, en el contraste y reevaluación de sus prácticas, experimentar la particular belleza en el vértigo de cada clase, entendiendo, junto a Greene, que: "No habrá final para nosotros en este recorrido, no puede haberlo" (Citado en Larrosa, 1995, p. 86).

\section{Hacia la experiencia estética en la enseñanza: oberturas}

La experiencia pedagógica muestra entonces sus extensiones desde las ideas y propuestas con que inician las acciones en la escuela. No obstante, aún no se ha señalado la experiencia que realmente contiene las mayores cargas e implicaciones, a saber, la que se vive en el ejercicio mismo de la clase, integrada al proceso de narración de los diarios, en una labor que, en este proyecto, se propuso para registrar las vivencias y variaciones de los vínculos entre música, poesía, expresión, evocación, literatura y escritura.

Se debe recordar que en el proceso propuesto resulta fundamental el vínculo con la música, como tejido que convoca la circulación de una experiencia estética en el aula; así, cada sesión se ha organizado en torno a un tema ligado al currículo, y se extrapola hacia las posibilidades de la conversación enfocada en el arte. Cada experiencia tiene un capítulo en los diarios y recorre distintas partes, que fluyen a través de aquellas órbitas que se quiso ilustrar en la cartografía del bosquejo de acción.

Nada más iniciar la clase con la conexión de equipos, los cuales fueron aportados por el maestro, se ven los primeros destellos y N..., una estudiante curiosa, pero en ocasiones también apática, pregunta por la música que sonará en el parlante, tal vez Alkolirycoz, grupo de Hip Hop del que ya habíamos hablado y cuya mención nos lleva a cantar a coro el estribillo de su canción El ritual.

Aún sin un arranque concreto, existe allí un gesto por atesorar, la sencilla alegría de coincidir en una canción y cantar los coros en compañía, generando un espacio de inclusión espontánea para estudiantes y maestro. Afortunadamente, no será la última vez que suceda; en otra sesión, D..., también estudiante, acompañará con su voz el recuerdo de la ranchera Sombras, de Javier Solís, luego de reflexionar sobre los versos que atraviesan Plantación adentro, de Rubén Blades, tema incorporado en la elasticidad elíptica del esquema propuesto para la clase dedicada a la literatura de la Conquista y la Colonia en América.

En sesiones posteriores, E..., un estudiante to-talmente distante y desinteresado respecto a cualquier asunto académico del colegio, sale de su ensimismamiento y responde al coro de Las tumbas, de Ismael Rivera, ritmo de salsa que se viene a integrar al enlazamiento de referencias musicales. La emoción de encontrar una respuesta de algún tipo en el estudiante inunda la sala, impregnando también a sus compañeros, quienes probablemente también sintieron la onda expansiva de tal reacción. Tal vez considerando condiciones como estas Gadamer planteó su inquietud sobre qué es hacer música:

En primera instancia: cantar [...] En ninguno de los casos significa que se interpretan las palabras que en ellos [los cantos] se pronuncian, sino que se interpreta el conjunto que las hace sonar y escuchar. 
Es algo increíble de la posibilidad de escuchar que todos conocemos. Yo lo llamo abrirse a algo (Citado en Schröder y Breuninger, 2005, p. 20).

El primer subrayado de la cita permite destacar una primera característica del canto: nunca se limita a una simple retahíla de palabras; se extiende en la resonancia generada en conjunto, que configura una apertura en medio de una experiencia ya proclive a la inscripción en lo estético, remitida incluso a un territorio que va más allá de lo racional.

Sonidos y canciones de distintos ritmos y géneros empiezan a recorrer las clases. Entre ellos aparece Atahualpa Yupanqui y su canción Camino del indio, que entra en contacto con el tema de literatura prehispánica. Luego de momentos de interacción y participación, donde los estudiantes tienen espacio para precisar sus impresiones y sensaciones, también surgen declaraciones que hacen visible la contraparte de estos ejercicios: La experiencia incluye resistencias.

K... declara que la música le produce sueño, lo cual revela su aburrimiento. Su apreciación permite acercarse a la importancia de reconocer la necesidad cuestionar constantemente los propios métodos $\mathrm{y}$ alejarse de algunas ideas ingenuas, que llevan a creer en fórmulas o modelos mágicos aplicables sistemáticamente para lograr resultados correctos. No existe un deux ex machina cuando hablamos de pedagogía, no es posible atribuir a un solo elemento un carácter mesiánico para el desarrollo de una práctica. Sea cual fuere la propuesta pedagógica, el despliegue heurístico y la construcción reflexiva siguen siendo el soporte al momento de actuar, derivando siempre en estado de alerta.

\section{El extrañamiento y el otro musicalizado}

En las variaciones de estas experiencias también se encuentra al otro. La otredad se revela de muchas maneras frente a la eventual posición propia como sujetos, y en los intercambios que van ocurriendo con los demás se hace evidente la complejidad de acercarse a ese otro. Empero, también se descubre, poco a poco, que la práctica pedagógica busca la posibilidad de esclarecimiento para esa comunicación vital. La música ha sido convocada en estas experiencias y acude, de cierta forma, como lo indica Baremboim:

La música siempre hace de contrapunto en el sentido filosófico de la palabra. Incluso cuando es lineal, siempre coexisten elementos contrarios, a veces incluso en conflicto unos con otros. La música en todo momento acepta comentarios de una voz a la otra, y tolera los acompañamientos subversivos como una antípoda necesaria para las voces principales. En la música coexisten en todo momento el conflicto, la negación y el compromiso (2008, p. 29).

El cauce conduce a una noción importante dentro de las categorías que surgen en estas experiencias: el extrañamiento; que hace parte del encuentro con lo diferente. El corpus musical incluido en la propuesta también configura un experimento en el que se encontrarán distintos ritmos, acaso extraños y distintos a los usuales para los jóvenes estudiantes. En el extenso mundo de la música se halla esa capacidad de plantear la coexistencia de contrarios y la conversación constante de la diversidad. Esta forma de extrañamiento, que no es la única, permite también encontrar un punto de partida para construir lo que aquí se llamado "apertura". 
Allí, entre las traslaciones de la propuesta pedagógica, en determinado momento se concretan algunas composiciones creativas escritas por los estudiantes, identificadas en el periastro de nuestra cartografía (Ver Figura 1). Dichas obras hacen parte de todo el recorrido experiencial iniciado desde el contacto con la música y con el pretexto planteado por los temas o ejes temáticos. De esta manera, los textos hacen parte de las revelaciones sobre las experiencias de clase y permiten percibir la forma en que se establece la conexión entre escuchar, sentir, elaborar y narrar los sentimientos, leer y, finalmente, escribir. Una conjunción verbal que convoca de manera integral lo que realmente trasciende en el aprendizaje de una persona en formación.

Los textos llevan a apreciar los tonos de la expresión de una experiencia sensible, vinculada con los ejercicios realizados y, por ello, justamente muchos de los constructos creados apelan a la apertura que se desprende de un extrañamiento inicial. Es posible reconocer este movimiento en las propias palabras de los estudiantes, revisadas mediante un análisis de sus composiciones que, como premisa, seguían el estilo de una crónica histórica regida por el uso de sus propios términos. Así, A..., describe la forma en que imagina navegar hacia otros lugares y el contacto con otras tribus; menciona que los extraños serán quienes cambien el futuro. Por su parte, V..., postula que las personas de distintas tribus empiezan a familiarizarse, mientras que S... exclama: "¿Qué grande era la curiosidad y qué pequeño es el mundo!”.

Enfrentar algo distinto en las construcciones narrativas no es un conflicto llano; el asunto se inclina hacía el reconocimiento del otro, la apertura hacia él e incluso hacia su conciliación, principio que defendía Hegel cuando buscó definir los mismos fines estéticos, reconociendo que su interés era que la oposición se disolviera a favor del espíritu humano, buscar la reconciliación, es decir, algo más profundo, donde las diferencias estén dispuestas en armonía.

\section{Oportunidad polifónica}

Como se ha visto, el espectro de la experiencia estética, generado en estos espacios pedagógicos, integra, desde la empatía espontánea, pasando por las actitudes de resistencia, hasta actividades habituales como la producción de textos; en todas ellas aviva el componente personal, sensible y aprendiente del ser humano que atraviesa las aguas de la escuela.

La unión de música y literatura permite alcanzar una flexibilidad que llega a horizontes de gran calado e interés para la educación, facilitando incluso el atrevimiento de la creación musical y la expresión danzada. En el contexto de la literatura de la Conquista y la Colonia introdujimos el ritmo de la salsa, con canciones de Cheo Feliciano y Rubén Blades. Tal mediación dio pie para incorporar instrumentos propios de este ritmo e intentar una orquestación, más lúdica y de exploración que otra cosa, pues no se trataba de ser un intérprete adecuado, sino de probar y sentir el contacto con la emisión, la vibración de la música. El disparate de tocar un instrumento sin formación previa, la descoordinación e incluso el ruido, tenían un lugar y un propósito, se trataba de un intento en conjunto, personas que buscaban acercarse a la literatura por distintos caminos artísticos, creando un espacio para las posibilidades de la locura y la fiesta en el acto educativo. 
En tal sentido, vale la pena recordar las valiosas consideraciones de Larrosa (2006) frente al concepto de la experiencia, que empiezan por una definición sencilla, pero de gran alcance: la experiencia es aquello que "me pasa"; no se trata de algo que simplemente ocurre y se desliza por la periferia, sino de un suceso que nos atraviesa y nos implica como seres humanos, así la cuestión de la experiencia "tiene muchas posibilidades en el campo educativo y posee enormes posibilidades teóricas, críticas y prácticas, cuando se le da una orientación adecuada" (Larrosa, 2006, pp. 88).

Resulta claro entonces que la intención de la propuesta pedagógica, expuesta someramente en el presente texto, se halla justamente en propiciar escenarios donde la experiencia orientada abre territorios de exploración, comunicación y prácticas educativas cada vez más formadoras y profundas. Junto a Larrosa (2006), se reconoce que suscitar experiencias estéticas en el proceso de enseñanza, resulta ser algo que "Nos forma (o nos de-forma o nos trans-forma), algo que nos constituye o nos pone en cuestión en aquello que somos" (p. 96); en este camino, se pone en marcha el latido de nuestras utopías, ofreciendo el panorama de un propósito, donde la escuela recobra su sentido como agente vital en los pasos estudiantiles, como territorio insuflado con un alma que acompaña las tonalidades de la niñez y la juventud.

\section{Conclusiones: conjuntos dinámicos dentro de las experiencias}

Es importante destacar algunos fenómenos manifestados en los procesos desarrollados, entre ellos, es posible concluir que, desde la sensibilidad misma, facilitada en las experiencias, los estudiantes fluctúan entre los sentimientos más difíciles de tramitar, desde la soledad, el abandono o el miedo, hasta la probabilidad de empatía con quien es totalmente diferente. A lo largo de las observaciones realizadas se encontró que, a pesar de tratarse de categorías que se pueden catalogar en forma independiente, existe continuidad, un entramado estrecho entre el conjunto: Extrañamiento(Apertura-Transformación)-Sentido ético.

Muchos de los procesos que surgen en las experiencias estéticas orbitan hacia la evocación del pasado. Al punto de que es posible postular la apropiación de una mirada nostálgica en el acto educativo, pues aquellas retrospectivas temporales activan también una apertura reflexiva y revitalizan el compromiso íntimo del estudiante con el aprendizaje. Así nace una categoría no considerada particularmente al inicio de los devenires de la propuesta, pero que participa decisivamente en los procesos de aprendizaje: la memoria; esfera que juega un rol sustancial en otro conjunto dinámico de estas experiencias pedagógicas: Memoria(Nostalgia-Apertura)-Identidad-Sentido ético.

La vinculación poética, musical, como experiencia estética de las actividades en el aula, configura una actitud y una mirada que cultiva la necesaria transgresión de tantas barreras petrificadas en la escuela. La escuela demanda vibraciones, un movimiento que halle ritmo, la polifonía espontánea que orqueste su libertad. ⿷匚 


\section{Referencias}

Bárcena, F. (2000). El aprendizaje como acontecimiento ético. Sobre las formas del aprender. Enrahonar, No. 31, pp. 9-33. Madrid: Departamento de teoría e historia de la educación, Universidad Complutense de Madrid.

Barenboim, D. (2008). El sonido es vida. El poder de la música. Bogotá: Norma.

Bloom, H. (1995). El canon occidental. Barcelona: Anagrama.

Larrosa, J. (1995). Déjame que te cuente. Ensayos sobre narrativa y educación. Barcelona: Laertes.

Larrosa, J. (2006). Sobre la experiencia. Aloma. Revista de psicología, ciènces de l'educació $i$ de l'esport, No. 19, pp. 87-112.

Lomas, C., y Jurado, F. (2015). Los libros de texto: ¿Tradición o innovación? Bogotá: Magisterio.

Max Neef, M. (1992). De la esterilidad de la certeza a la fecundidad de la incertidumbre. Obtenido desde https://docplayer.es/88192540-De-laesterilidad-de-la-certeza-a-la-fecundidad-de-laincertidumbre-1-manfred-max-neef-premionobel-alternativo-chile.html

Schröder, G., y Breuninger, H. (2005). Teoría de la cultura. Buenos Aires: FCE. 